\title{
200 años de guerra y paz en Colombia: números y rasgos estilizados*
}

Recibido: 04/05/2019 | Revisado: 14/06/2019 | Aceptado: 19/06/2019

DOI: 10.17230/co-herencia.16.31.11

\author{
Jorge Giraldo Ramírez ${ }^{* *}$ \\ jorgegiraldo@eafit.edu.co \\ Jose Antonio Fortou ${ }^{* * *}$ \\ jfortour@eafit.edu.co \\ María Paulina Gómez Caicedo*** \\ mgomez25@eafit.edu.co
}

\begin{abstract}
Resumen Este artículo presenta las conclusiones de un estudio cuantitativo y longitudinal sobre las guerras civiles colombianas, desde el momento de la constitución del país como unidad política estable, y de las formas como se accedió a la paz después de cada una de ellas. Incluye, además, una comparación cuantitativa con los demás países de Hispanoamérica. Se concluye que hay diferencias críticas entre las guerras del siglo XIX y las del xx, en cuanto a duración y severidad, así como en cuanto a las formas de la paz (victorias y amnistías o acuerdos negociados), con excepción de la Guerra de Los Mil Días.
\end{abstract}

\section{Palabras clave:}

Guerra civil, paz, acuerdos de paz, independencias, historia de Colombia.

\section{0 years of war and peace in Colombia: numbers and stylized features}

\begin{abstract}
The This paper presents the findings of a quantitative and longitudinal study both about the Colombian civil wars, from the moment the country formed as a stable political unit, and about the ways in which peace was achieved after each of them. It also provides a quantitative comparison with the other Latin American countries. The paper concludes that there are critical differences between the wars of the $\mathrm{XIX}$ century and those of the XX, in terms of duration and harshness, as well as in the forms of peace (victories and amnesties or negotiated agreements), with the exception of the Thousand Days' War.
\end{abstract}

\section{Keywords:}

Civil war, peace, peace agreements, independence processes, history of Colombia.
Este artículo es producto del trabajo del grupo de investigación Sociedad, Política e Historias Conectadas, de la Universidad EAFIT.

** Doctor en Filosofía y profesor de la Universidad EAFIT, Medellín, Colombia.

*** Doctor en Ciencia Política y profesor de la Universidad EAFIT, Medellín, Colombia. ORCID: 0000-00023127-4288

*****a Politóloga de la Universidad EAFIT, Medellín, Colombia. 
Los años 2010 y 2019 enmarcan el rango de las celebraciones del bicentenario de la Independencia de la Nueva Granada y han traído consigo el resurgimiento de los exámenes sobre la trayectoria del país a larga duración. A propósito del primer hito, se presenta un trabajo cuantitativo y longitudinal, con el cual se intenta animar la discusión en torno a las guerras civiles colombianas (Giraldo y Fortou, 2011). Allí, se describen estas guerras a partir de una serie de cifras, con énfasis en el número de muertes en combate (severidad), la duración de dichos conflictos, su intensidad y el tamaño de los poderes armados enfrentados. El análisis indicó diferencias importantes entre las guerras decimonónicas y las posteriores.

El cierre de las efemérides, en 2019 -tres años después de suscrito el acuerdo entre el Estado colombiano y las FARC-, supone una oportunidad para actualizar aquel estudio, ya no solo en clave de guerra, sino también de paz. Así las cosas, este artículo pretende retomar los hallazgos de hace nueve años e incluir los procesos de paz adelantados en Colombia desde el siglo XIX, en la misma clave descriptiva, pero incluyendo nuevos elementos interpretativos. Tal y como entonces, se señalan las diferencias entre las guerras del siglo XIX y las del XX, argumentando que estas diferencias también se evidencian en cómo se ha hecho la paz.

Como punto de partida, se retoma la caracterización de las guerras civiles en Colombia e Hispanoamérica, en términos de duración. Adicionalmente, se muestra la manera en que ha cambiado la capacidad del Estado colombiano después de las guerras civiles. Posteriormente, se introduce una mirada a las formas en que se ha hecho la paz a lo largo de la historia republicana del país. Se sugiere que hay diferencias claves entre el siglo XIX y los siglos XX y XXI, las cuales se expresan tanto en la guerra, propiamente, como en la paz. 


\section{Guerra}

En esta sección, se presentan algunas cifras que permiten alcanzar dos objetivos. En primer lugar, se ubican las guerras civiles colombianas en el contexto hispanoamericano desde las independencias. En segundo lugar, se caracterizan dichas guerras según criterios de severidad, poder militar y su relación con la construcción del Estado en Colombia. La estatalidad en escenarios de posconflicto (Fukuyama, 2005) permite hacer el giro hacia el estudio de cómo se ha hecho la paz en el país.

Para comenzar esta revisión, se presenta una mirada comparada con Hispanoamérica. Esto permite relativizar y contextualizar las guerras civiles colombianas, pues este no es el único país de la región con una larga historia de conflicto y violencia internos. La Tabla 1 muestra el número de guerras intraestatales ocurridas en los distintos países de Hispanoamérica, según el Correlates of War Project para el período bicentenario (desde 1832). Para este proyecto (Sarkees y Wayman, 2010), las guerras intraestatales son disputas armadas entre dos bandos organizados al interior de un Estado (Gobierno central y un grupo armado no estatal). Esta disputa debe ser por el control del Gobierno, o por problemas regionales o locales, y se expresa violentamente de forma masiva, con un mínimo de 1000 muertes en un año.

La revisión de estos datos muestra que el caso colombiano tiene elementos comparables con los del resto del continente. Por un lado, en el país han ocurrido guerras a la misma razón que en Argentina o México, lo cual matiza su excepcionalidad. Por otro lado, la duración promedio de las guerras civiles colombianas es la mayor de Hispanoamérica; esto se debe a la duración excepcional de las dos últimas guerras: La Violencia y el conflicto armado. En general, de las comparaciones internacionales puede deducirse que, a pesar de su recurrencia y duración, las guerras civiles colombianas, típicamente, han sido menos severas (total de muertes en combate) e intensas (muertes en combate por año). 
Tabla 1. Guerras civiles en Hispanoamérica, 1832-2007

\begin{tabular}{|l|c|c|}
\hline \multicolumn{1}{|c|}{ País } & Cantidad & $\begin{array}{c}\text { Duración mediana } \\
\text { (meses) }\end{array}$ \\
\hline Argentina & 10 & 3 \\
\hline Bolivia & 3 & 0 \\
\hline Chile & 3 & 3 \\
\hline Colombia & 10 & 15 \\
\hline Costa Rica & 1 & 1 \\
\hline Ecuador & 2 & 9 \\
\hline El Salvador & 2 & 3,5 \\
\hline Guatemala & 3 & 10 \\
\hline Honduras & 1 & 1 \\
\hline México & 13 & 6 \\
\hline Nicaragua & 2 & 5,5 \\
\hline Perú & 6 & 3 \\
\hline Paraguay & 2 & 7 \\
\hline Uruguay & 1 & 8 \\
\hline Venezuela & 5 & 6 \\
\hline
\end{tabular}

Fuente: elaboración propia con base en Correlates of War Intra-State Wars Dataset, versión 4.1 (Sarkees y Wayman, 2010), y Giraldo y Fortou (2011).

En el caso específico de Colombia, se identificaron diez guerras civiles desde la creación de la República de la Nueva Granada, en 1832, como la unidad política que perduró hasta hoy bajo distintas denominaciones (Giraldo y Fortou, 2011). La Tabla 2 ofrece un listado de estas guerras, acompañado de algunas de sus principales características: fechas de inicio y fin, la severidad o costo humano, y el tamaño estimado de los poderes armados en disputa, con una actualización de las cifras. Se concluye que las diez guerras duraron,

1 La metodología está detallada en Giraldo y Fortou (2011). Aquí, es preciso evidenciar la diversidad de las fuentes de información utilizadas, según la guerra civil: Supremos (Gaitán, 1995; Ortiz, 2004; Zuluaga, 1998); 1851 (Camacho, 1971; Gaitán, 1995; Safford y Palacios, 2002); Artesanos (Camacho, 2018; Gaitán, 1995; De Mosquera, 1982; Safford 
en conjunto, 571 meses (casi 48 años), de un total de 184 años de historia republicana, es decir, un $26 \%$ del tiempo total (Giraldo y Fortou, 2011). ${ }^{2}$ En este caso, en vez de dar una cifra única de severidad y ejércitos totales durante las diez guerras civiles identificadas en dicho artículo, se ofrece un rango, delimitado por la cifras mínima y máxima provistas por las fuentes para cada guerra. De esta manera, se incorporan críticas metodológicas, como la de Malcolm Deas (2018).

Tabla 2. Guerras civiles en Colombia: severidad y tamaño de los ejércitos

\begin{tabular}{|l|r|r|r|r|r|r|}
\hline \multicolumn{1}{|c|}{ Guerra } & Inicio & Fin & $\begin{array}{c}\text { Mínimo } \\
\text { muertes }\end{array}$ & $\begin{array}{c}\text { Máximo } \\
\text { muertes }\end{array}$ & $\begin{array}{c}\text { Mínimo } \\
\text { ejércitos }\end{array}$ & $\begin{array}{c}\text { Máximo } \\
\text { ejércitos }\end{array}$ \\
\hline Supremos & 1839 & 1841 & 1000 & 3366 & 2900 & 3000 \\
\hline 1851 & 1851 & 1851 & 300 & 1000 & 1500 & 10000 \\
\hline Artesanos3 & 1854 & 1854 & 2000 & 2000 & 400 & 14000 \\
\hline Soberanías & 1860 & 1862 & 1000 & 6000 & 2000 & 13800 \\
\hline Escuelas & 1876 & 1877 & 3870 & 10000 & 1000 & 40000 \\
\hline 1885 & 1885 & 1885 & 1000 & 3000 & 2500 & 11200 \\
\hline 1895 & 1895 & 1895 & 1200 & 2000 & 5000 & 22000 \\
\hline Mil Días & 1899 & 1902 & 25000 & 170000 & 8182 & 105000 \\
\hline Violencia & 1946 & 1957 & 6000 & 145340 & 17000 & 49400 \\
\hline Conflicto armado & 1994 & 2005 & 14504 & 125243 & 153791 & 207000 \\
\hline
\end{tabular}

Fuente: elaboración propia con base en Giraldo y Fortou (2011).

y Palacios, 2002; Tirado, 2001; Zambrano, 1998); Soberanías (Gaitán, 1995; Gutiérrez, 2018; Quijano, 1982); Escuelas (Gaitán, 1995; Ortiz, 2004; 2018); 1885 (Deas, 1993; 2018; Gaitán, 1995; Palacio, 1936; Ortiz, 2004); 1895 (Aguirre, 1997; Deas, 2018; Gaitán, 1995); Mil Días (Escobar, 2018; Gaitán, 1995; Martínez, 1956; Meisel y Romero, 2017; Otero, 2007; Pardo, 2004; Plazas, 1985; Valencia, 1989; Villegas y Yunis, 1978); La Violencia (Blair, 1993; Gaitán, 1995; Oquist, 1978; Palacios, 2002; Ramsay, 2000; Santos, 2007); Conflicto armado (Otero, 2007; 2010; Pettersson, Högbladh y Öberg, 2019; Sánchez, Díaz y Formisano, 2007); Sarkees y Wayman (2010) es fuente para todas las guerras.

2 Para La Violencia y el conflicto, es más difícil establecer inicios y finales precisos. En este ejercicio, se escogieron los períodos que coinciden con los momentos de mayor severidad. Una discusión más extensa se encuentra en Giraldo y Fortou (2011).

3 También conocida como el golpe de Melo o la Guerra Artesano-Militar (Uribe y López, 2006). Uribe de Hincapié y López Lopera muestran que se trató de una guerra civil entre dos bandos: el general José María Melo y los artesanos, contra una alianza de los liberales radicales y conservadores. 
Para el caso de las guerras del siglo xx, estas fueron contiendas más largas que las decimonónicas. En general, fueron más severas (más víctimas), con la excepción importante de la Guerra de los Mil Días. Si bien hubo elementos notables de guerra irregular en varias de las guerras del siglo XIX, ${ }^{4}$ el tipo de confrontación dominante (no único) fue de tipo convencional. La Violencia y El Conflicto, en cambio, se libraron principalmente como guerra irregular o guerra de guerrillas.

Una diferencia adicional estriba en que las victorias bloquearon el fortalecimiento de las capacidades estatales, mientras que las negociaciones le abrieron camino a la construcción del Estado. Después de cada guerra, el Ejército nacional se vio disminuido, bajó el gasto militar, se permitió el armamento de civiles, se mantuvieron las prerrogativas regionales frente al poder central, el Estado no pudo aumentar su capacidad fiscal y se prolongó la debilidad del aparato administrativo. Estas tendencias cambiaron desde 1990, en coincidencia con los primeros acuerdos para resolver el conflicto armado. La Figura 1 muestra los cambios en la capacidad estatal en Colombia desde 1830. En este caso, se utilizó el indicador compuesto de capacidades nacionales desarrollado por Correlates of War (Singer, 1987), el cual conceptualiza la capacidad estatal de manera similar a la noción de poder infraestructural en Mann (1997a; 1997b; 2007) e incluye medidas de capacidad coercitiva, producción industrial y urbanización. La línea sólida muestra los cambios en el indicador, mientras que las barras grises indican períodos de guerra civil.

Efectivamente, los datos indican un mayor crecimiento de la capacidad estatal tras las guerras civiles del siglo xx. Además, en el siglo XIX, las élites de ambos partidos tenían un fuerte sesgo antimilitar, evidenciado en el desmonte constante del Ejército (Deas, 2018; Moreno, 2014). ${ }^{5}$ Por ejemplo, varias fuentes coinciden en que, tras la derrota del general Melo en la Guerra Artesano-Militar de

4 En los apéndices del libro de Uribe de Hincapié y López Lopera (2006), se evidencia la cantidad de actores armados que constituían guerrillas en las guerras de los Supremos (1851 y 1854). Orozco Abad (2006) ofrece una discusión sobre el papel de los rebeldes colombianos, incluyendo guerrilleros, como combatientes durante el siglo XIX.

5 En particular, la ideología política de liberales radicales, quienes, desde una posición de hegemonía política desde mediados del siglo xix hasta 1885 y con figuras como Florentino González, plantearon como propósito disminuir el Ejército (Tamayo, 2017). 
1854, el Ejército central fue reducido drásticamente, quedando solo entre 400 y 588 efectivos (Camacho, 2018; Tirado, 2001; Safford y Palacios, 2002). En contraste, Blair (1993) estima un crecimiento del Ejército tras La Violencia, y Otero Prada (2007) coincide con la apreciación de un fortalecimiento de la capacidad coercitiva del Estado hacia el siglo XXI y el final del conflicto armado. Así, pese a la impresión de continuidad dada por el número de guerras civiles en el país, hay importantes rupturas.

Figura 1. Capacidades materiales nacionales y guerras civiles, $1830-2012$

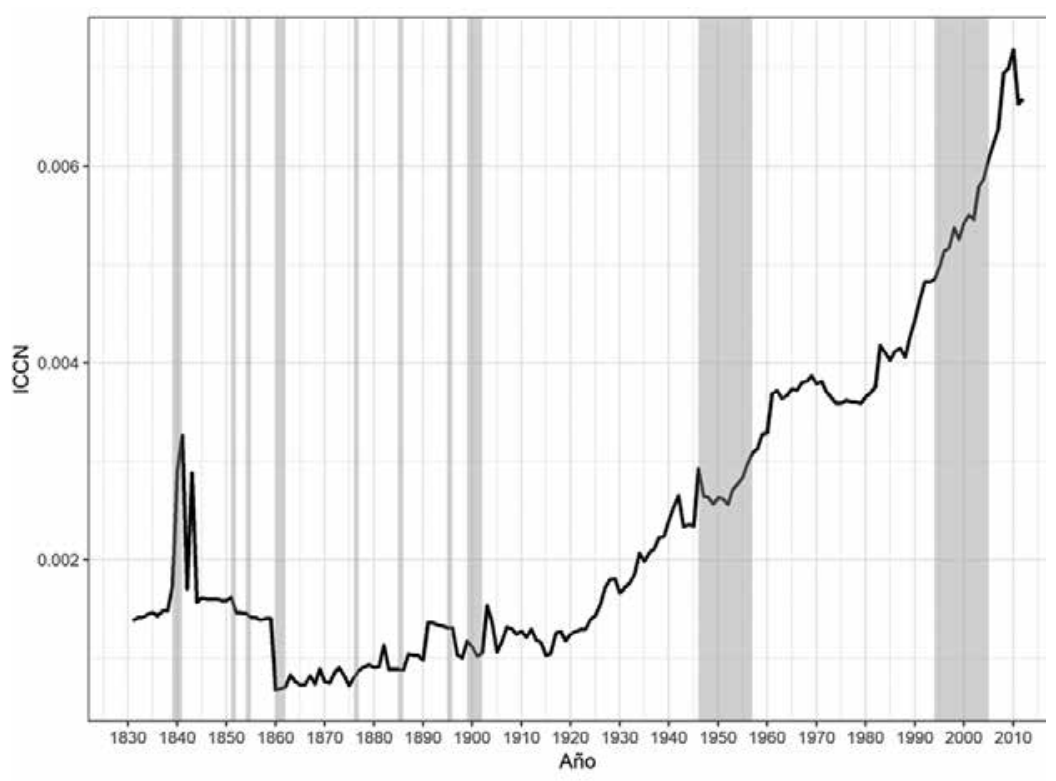

Fuente: elaboración propia con base en Correlates of War National Material Capabilities Dataset, version 5.0 (Singer, 1987).

\section{Paz}

La recurrencia de la guerra en Colombia ha traído consigo el hábito de hacer la paz. Esta es una característica histórica, una tradición siempre disponible cada vez que los gobernantes y los partidos políticos se vieron abocados a lidiar con la oposición armada. Sin 
embargo, la paz se ha construido por distintas vías: victoria, impotencia, negociación.

Durante el siglo XIX la principal vía de acceso a la paz fue la victoria militar. Incluso, en casos como los de las guerras ArtesanoMilitar y la de las Soberanías, ${ }^{6}$ con el triunfo del bando opositor. También hubo uso de acuerdos y negociaciones, esponsiones, ${ }^{7}$ indultos y olvidos (Camacho, Garrido y Gutiérrez, 2018; Uribe y López, 2008a; 2008b), además de otras prácticas y discursos pacifistas (Cardona y Céspedes, 2017; Pinzón, 2017).

Más sólida y antigua que la negociación, ha sido la tradición del perdón y la amnistía. Aguilera Peña (2012) inventarió cerca de dos centenares de amnistías e indultos, y los agrupó en grandes eventos, de los cuales seis ocurrieron en la etapa republicana. A ellos habría que agregar las amnistías masivas del siglo XXI que cobijaron a más de cincuenta mil combatientes. No faltaron los choques entre quienes propugnaban por la "destrucción de las facciones levantadas en armas”, como José Ignacio de Márquez (1793-1880), y quienes, como Rafael Núñez (1825-1894), predicaron amplio perdón y clemencia (Garrido, 2019). Esta confrontación sigue lozana en el siglo XXI, entre los partidarios de una justicia retributiva más o menos fuerte, como condición necesaria para el tratado de paz, y los que defienden una visión más magnánima y generosa hacia los antiguos guerreros.

La Tabla 3 evidencia esta historia. Para la recopilación de las amnistías se utilizaron dos fuentes principales: las guerras registradas en Correlates of War y el artículo de Aguilera Peña (2012), que destaca, de entre 200 momentos de amnistía e indultos en la historia republicana de Colombia, siete en los que se otorgaron perdones no solo a los delincuentes políticos, sino también a los comunes. Partiendo de ambas fuentes, se emparejaron los beneficios jurídicos con su res-

6 Esta denominación, la de guerra civil de o por las Soberanías, viene de Uribe de Hincapié y Lopera (2008a).

7 En varios capítulos, los contribuyentes al libro Paz en la República resaltan el uso de estos instrumentos. Para una revisión amplia de las aproximaciones a la paz tras las guerras civiles del siglo XIX, ver la introducción a dicho texto (Camacho, Garrido y Gutiérrez, 2018). En particular, las esponsiones -un tipo de armisticio-fueron una figura recurrente; Uribe de Hincapié (2011), y Uribe de Hincapié y López Lopera (2008a) afirman su papel central en el siglo XIX, y destacan eventos como la esponsión de los Árboles (1840) y la de Manizales (1860). El texto de esta última puede consultarse en "La Esponsión de Manizales" (1989), en la revista Análisis Político. 
pectiva guerra, y se registró, también, el mecanismo legal utilizado por el Gobierno de la época para cumplir la concesión de perdones a los delincuentes políticos y comunes. Esta información muestra la prevalencia de las amnistías y perdones en el siglo XIX.

Tabla 3. Paces, acuerdos y amnistías tras las guerras civiles en Colombia

\begin{tabular}{|c|c|c|}
\hline Guerra & Terminación & Instrumentos y disposiciones \\
\hline Supremos & Victoria & $\begin{array}{l}\text { Amnistía para delitos políticos y comunes } \\
\text { cometidos hasta } 1843 \text {, expedida como } \\
\text { decreto por Tomás Cipriano de Mosquera, } \\
\text { en } 1849 \text {. } \\
\text { Constitución de } 1843 \text {. }\end{array}$ \\
\hline 1851 & Victoria & $\begin{array}{l}\text { Amnistía general de } 1853 \text {, siguiendo una } \\
\text { secuencia de indultos en 1851-1853. } \\
\text { Constitución de } 1853 \text {. }\end{array}$ \\
\hline Artesanos & Victoria & $\begin{array}{l}\text { Indultos para terminar la guerra en } 1854 \text { - } \\
1855 . \\
\text { Constitución de } 1858 \text {. }\end{array}$ \\
\hline Soberanías & Victoria & $\begin{array}{l}\text { Acto presidencial de Tomás Cipriano } \\
\text { Mosquera para la liberación de delincuentes } \\
\text { políticos y comunes, por medio del Artículo } \\
3 \text { del Pacto Transitorio del } 20 \text { de septiembre } \\
\text { de } 1861 \text {. } \\
\text { "Arreglo" aprobado por Manuel Murillo } \\
\text { Toro, en } 1864, \text { llevó a la entrega de armas y } \\
\text { amnistía de las fuerzas antioqueñas. } \\
\text { Constitución de } 1863 \text {. }\end{array}$ \\
\hline $\begin{array}{l}\text { Escuelas, } \\
1885 \text { y } 1895\end{array}$ & Victorias & $\begin{array}{l}\text { Prescripción de la pena para militares que, al } \\
\text { servicio del gobierno o la revolución, hayan } \\
\text { cometido delitos comunes o políticos, según } \\
\text { Ley } 27 \text { de } 1907 \text { (derogada por la Ley } 4 \text { de } \\
\text { 1908). } \\
\text { Constitución de } 1886 \text {. }\end{array}$ \\
\hline Mil Días & Negociación & $\begin{array}{l}\text { Acuerdos de Neerlandia y USS Wisconsin. } \\
\text { Perdones por iniciativa del gobiernode Reyes } \\
\text { a todos los colombianos comprometidos en } \\
\text { la revolución armada, exceptuando los que } \\
\text { incurrieron en delitos comunes, a través de } \\
\text { Decreto } 933 \text { de } 1902 \text { y Ley } 57 \text { de } 1903 \text {. }\end{array}$ \\
\hline
\end{tabular}




\begin{tabular}{|l|l|l|}
\hline \multicolumn{1}{|c|}{ Guerra } & Terminación & \multicolumn{1}{c|}{ Instrumentos y disposiciones } \\
\hline Violencia & Negociación & $\begin{array}{l}\text { Pactos de Benidorm (1956) y Sitges (1957) } \\
\text { ponen fin al conflicto entre las dos partes } \\
\text { principales. } \\
\text { Amnistía dictada por Rojas Pinilla a delitos } \\
\text { políticos cometidos antes del 1. }{ }^{\circ} \text { de enero } \\
\text { de 1954, por medio de los Decretos 1823 de } \\
1954, \text { y 250 de 1958. }\end{array}$ \\
\hline $\begin{array}{l}\text { Conflicto } \\
\text { armado }\end{array}$ & Negociación & $\begin{array}{l}\text { Hasta 22 acuerdos en el período 1975-2018. } \\
\text { Acuerdo final en 2016 con las FARC. }\end{array}$ \\
\hline
\end{tabular}

Fuente: elaboración propia con base en Aguilera (2012).

Las guerras del siglo $\mathrm{xx}$, en cambio, no terminaron con victorias militares clásicas, sino que lo hicieron mediante negociaciones, casi siempre fraccionadas en pactos múltiples. La Guerra de los Mil Días, al menos con tres pactos (Neerlandia, Wisconsin y Chinácota); y el conflicto armado -hasta el presente-, mediante diez acuerdos distintos de paz (seis con guerrillas, dos con paramilitares y dos con milicias urbanas). La curva nacional de aprendizaje de la negociación de paz no fue fácil. De hecho, la solución de la Guerra de los Mil Días y de La Violencia parece haber llegado de la mano del desgaste de los bandos, lo cual puede relacionarse con el concepto de "paz por la impotencia”, planteado por Aron (1985).

El caso de La Violencia es aún más claro. En primera instancia, el bloqueo mutuo de los partidos enfrentados y su incapacidad de controlar completamente las fuerzas activas en los campos condujeron a la solución de incluir un tercero: las fuerzas armadas, en cabeza del general Rojas Pinilla. Posteriormente, los pactos de Sitges y Benidorm se justificaron como solución para evitar la prolongación del Gobierno militar y como pacto entre los partidos. La doble transición, a la paz y a la democracia, llegó por la vía de la impotencia.

La Tabla 4 muestra el número de acuerdos de paz firmados en Hispanoamérica, desde 1975, según el Uppsala Conflict Data Program (Pettersson, Högbladh y Öberg, 2019). Pese al número limitado de conflictos durante este período en Colombia (uno), la cantidad de 
actores involucrados, junto con la duración del conflicto armado, han llevado a un alto número de acuerdos parciales (22 en total). A manera de comparación, en Colombia se han firmado más acuerdos que en Guatemala y El Salvador, y que en El Salvador, México y Nicaragua juntos.

Tabla 4. Acuerdos de paz en Hispanoamérica, 1975-2018

\begin{tabular}{|l|c|}
\hline \multicolumn{1}{|c|}{ País } & Número de acuerdos \\
\hline Colombia & 22 \\
\hline Guatemala & 16 \\
\hline El Salvador & 13 \\
\hline México & 6 \\
\hline Nicaragua & 2 \\
\hline
\end{tabular}

Fuente: elaboración propia con base en Uppsala Conflict Data Program Peace Agreement Dataset, versión 19.1 (Pettersson, Högbladh y Öberg, 2019).

Las negociaciones del conflicto armado, con excepción de la que terminó en 2016 con las FARC, fueron más fáciles que las anteriores de liberales y conservadores. Entre 1989 y 1997, se dieron siete procesos de negociación con distintos grupos insurgentes de izquierda, en una fase que puede denominarse "de paz consentida" (Giraldo, 2018). Fueron procesos breves, casi sumarios, que no suscitaron ninguna oposición significativa entre las fuerzas políticas y sociales del país, a pesar de que implicaron amnistías absolutas, participación política parlamentaria y en el poder ejecutivo, y que se dieron en convergencia con un proceso constituyente.

Otra diferencia crucial entre las paces del siglo XIX y las posteriores es la incorporación de los vencidos al ejercicio del poder político. Las paces del siglo XIX fueron excluyentes, y la representación política posterior fue acaparada por los vencedores. En este mismo sentido, las guerras de los Supremos, 1851, 1854, Soberanías y 1885 fueron prontamente seguidas por nuevas constituciones, en las que los vencedores intentaban imponer su modelo (Camacho, Garrido y Gutiérrez, 2018). En contraste, las reformas constitucionales del 
Frente Nacional y la Constitución de 1991 responden más a dinámicas de negociación: en el caso del Frente Nacional, a las "negociaciones de caballeros" de liberales y conservadores agotados (Wilde, 1982); y en el caso de 1991, a una convocatoria nacional amplia y más incluyente con representación de diversos sectores (Giraldo y Fortou, 2012; Uprimny, 2011).

Desde la finalización de la Guerra de los Mil Días hasta el presente, la paz ha sido incluyente, con variaciones de grado, y ha contemplado, expresamente, la participación institucional de las fuerzas políticas que antes habían apelado a las armas. Quizás esto explique que los períodos más largos de posconflicto se hayan dado en el siglo XX.

\section{Conclusiones}

Las guerras civiles han sido recurrentes en Colombia y cubren una cuarta parte del tiempo, desde la instauración de la república. La amnistía y el perdón, desde el siglo XIX, y la vía negociada de los conflictos armados, durante el xx, han sido rasgos idiosincrásicos de la vida política en el país. La nítida convergencia entre el Estado débil y la guerra civil recurrente invitan a pensar la paz en clave de construcción estatal I

\section{Bibliografía}

Aguilera Peña, M. (2012). Refundemos la nación: perdonemos a delincuentes y políticos comunes. Análisis Político, 25(76), 5-40.

Aguirre, M. (1997). Insurgencia urbana en Bogotá: Motín, conspiración y guerra civil, 18931895. Bogotá: Colcultura.

Aron, R. (1985). Paz y guerra entre naciones. Madrid: Alianza Editorial.

Blair, E. (1993). Las Fuerzas Armadas: Una mirada civil. Bogotá: CINEP.

Camacho Arango, C. (2018). Pero no basta vencer, 1854-1859. En C. Camacho Arango, M. Garrido Otoya y D. Gutiérrez Ardila (eds.), Paz en la República (pp. 115-151). Bogotá: Universidad Externado de Colombia.

Camacho Arango, C., Garrido Otoya, M. y Gutiérrez Ardila, D. (2018). Introducción. En Paz en la República (pp. 15-28). Bogotá: Universidad Externado de Colombia.

Camacho Roldán, S. (1971). Memorias. Medellín: Editorial Bedout. 
Cardona Z., P. y Céspedes, C. (2017). Cerbeleón Pinzón y la Paz Pública. Co-herencia, 14(26), $13-22$.

De Mosquera, T. C. (1982). Resumen de los acontecimientos que han tenido lugar en la República: Memoria de la guerra civil de 1854. Bogotá: Editorial Incunables.

Deas, M. (1993). Del poder y la gramática y otros ensayos sobre historia, política y literatura colombianas. Bogotá: Tercer Mundo.

Deas, M. (2018). Sobre la paz en el siglo xIX, con un examen particular de cómo terminaron las guerras de 1885 y 1895. En C. Camacho Arango, M. Garrido Otoya y D. Gutiérrez Ardila (eds.), Paz en la República (pp. 239-269). Bogotá: Universidad Externado de Colombia.

Escobar Guzmán, B. (2018). Tras la guerra de los Mil Días: hacia una paz duradera. En C. Camacho Arango, M. Garrido Otoya y D. Gutiérrez Ardila (eds.), Paz en la República (pp. 271-307). Bogotá: Universidad Externado de Colombia.

Fukuyama, F. (2005). Building Democracy After Conflict: 'Stateness' First. Journal of Democracy, 1, 84-88.

Gaitán Daza, F. (1995). Una indagación sobre las causas de la violencia en Colombia. En M. Deas y F. Gaitán Daza (eds.), Dos ensayos especulativos sobre la violencia en Colombia (pp. 89-415). Bogotá: Fonade/Departamento Nacional de Planeación.

Giraldo Ramírez, J. (2018). Colombia: así en la guerra como en la paz. Madrid: La Huerta Grande.

Giraldo Ramírez, J. y Fortou, J. A. (2011). Una comparación cuantitativa de las guerras civiles colombianas, 1830-2010. Análisis Político, 72, 3-21.

Giraldo Ramírez, J. y Fortou, J. A. (2012). Proceso y resultado: un balance de la constitución. En F. Cortés Rodas (ed.), Reinventar la democracia: Memorias (pp. 91-113). Medellín: Universidad de Antioquia.

Gutiérrez Ardila, D. (2018). Una paz plagada de guerras, 1863-1876. En C. Camacho Arango, M. Garrido Otoya y D. Gutiérrez Ardila (eds.), Paz en la República (pp. 153-194). Bogotá: Universidad Externado de Colombia.

La Esponsión de Manizales (1989). Análisis Político, 6, 123.

Mann, M. (1997a). Las fuentes del poder social (Vol. I). Madrid: Alianza Editorial.

Mann, M. (1997b). Las fuentes del poder social (Vol. II). Madrid: Alianza Editorial.

Mann, M. (2007). El poder autónomo del Estado: sus orígenes, mecanismos y resultados. Relaciones Internacionales, 5, 1-43.

Martínez Landínez, J. (1956). Historia militar de Colombia (Tomo I). Bogotá: Editorial Iqueima. 
Meisel, A. y Romero, J. E (2017). La mortalidad en la Guerra de los Mil Días, 1899-1902. Cartagena: Banco de la República.

Moreno Mancera, J. D. (2014). Relaciones cívico-militares en Colombia: supremacía y control de los partidos políticos sobre la organización militar. Revista Científica General José María Córdova, 12(13), 333-352.

Oquist, P. (1978). Violencia, conflicto y política en Colombia. Bogotá: Instituto de Estudios Colombianos/Biblioteca Banco Popular.

Orozco Abad, I. (2006). Combatientes, rebeldes y terroristas: Guerra y derecho en Colombia (2.. ed.). Bogotá: Editorial Temis.

Ortiz Mesa, L. J. (2004). Fusiles y plegarias: Guerra de guerrillas en Cundinamarca, Boyacá y Santander, 1876-1877. Medellín: La Carreta Editores.

Ortiz Mesa, L. J. (2018). De la paz que perdieron los radicales a la paz científica, 1876-1885. En C. Camacho Arango, M. Garrido Otoya y D. Gutiérrez Ardila (eds.), Paz en la República (pp. 195-238). Bogotá: Universidad Externado de Colombia.

Otero Prada, D. (2007). Las cifras del conflicto colombiano. Bogotá: INDEPAZ.

Otero Prada, D. (2010). Las muertes del conflicto colombiano en el período 1964-2008. Bogotá: Universidad Central.

Palacio, J. (1936). La guerra del 85. Bogotá: Editorial Cromos.

Palacios, M. (2002). El Café en Colombia, 1850 - 1970. Una historia económica, social y política. Bogotá: Editorial Planeta.

Pardo, R. (2004). La historia de las guerras. Barcelona: Ediciones B.

Pettersson, T., Högbladh, S. y Öberg, M. (2019). Organized Violence, 1989-2018 and Peace Agreements. Journal of Peace Research, 56(4), 1-15.

Pinzón, C. (2017). Sobre la Paz Pública. Co-herencia, 14(26), 23-56.

Plazas Olarte, G. (1985). La Guerra Civil de los Mil Días. Estudio militar. Tunja: Academia Boyacense de Historia.

Quijano Otero, J. M. (1982). Diario de la guerra civil de 1860 y otros sucesos políticos. Bogotá: Editorial Incunables.

Ramsay, R. (2000). Guerrilleros y soldados. Bogotá: Tercer Mundo Editores.

Safford, F. y Palacios, M. (2002). Colombia: País fragmentado, sociedad dividida. Bogotá: Norma.

Sánchez, F., Díaz, A. M. y Formisano, M. (2007). Conflicto, crimen violento y actividad en Colombia: un análisis espacial. En F. Sánchez (ed.), Las cuentas de la violencia: Ensayos económicos sobre el conflicto y el crimen en Colombia (pp. 63-130). Bogotá: Norma. 
Santos Pico, M. J. (2007). Historia militar del Ejército de Colombia. Bogotá: Centro de Estudios Históricos del Ejército.

Sarkees, M. R. y Wayman, F. (2010). Resort to War: 1816-2007. Washington, DC: CQ Press.

Singer, J. D. (1987). Reconstructing the Correlates of War Dataset on Material Capabilities of States, 1816-1985. International Interactions, 14, 115-132.

Tamayo Arboleda, F. L. (2017). Autoritarismo y liberalismo. Una mirada a partir de la obra de Florentino González a la ideología liberal en Colombia en el siglo xIx. Estudios Políticos, 51, 106-127.

Tirado Mejía, A. (2001). El Estado y la política en el siglo xIx. Bogotá: El Áncora Editores.

Uprimny, R. (18 de julio de 2011). La Constitución de 1991 en perspectiva (II). El Espectador.

Uribe de Hincapié, M. T. (2011). La filigrana de la paz: acuerdos, exponsiones, indultos y amnistías. En L. López Lopera (ed.), Un retrato fragmentado: Ensayos sobre la vida social, económica y política de Colombia, siglos xIX y xx (pp. 74-96). Medellín: La Carreta Editores.

Uribe de Hincapié, M. T. y López Lopera, L. M. (2006). Las palabras de la guerra: Un estudio sobre las memorias de las guerras civiles en Colombia. Medellín: La Carreta Editores.

Uribe de Hincapié, M. T. y López Lopera, L. M. (2008a). La Guerra por las Soberanías: Memorias y relatos en la guerra civil de 1859-1862 en Colombia. Medellín: La Carreta Editores.

Uribe de Hincapié, M. T. y López Lopera, L. M. (2008b). Los discursos del perdón y del castigo en la guerra civil colombiana de 1859-1862. Co-herencia, 5(8), 1-30.

Valencia Tovar, A. (1989). Historia militar contemporánea. En A. Tirado Mejía (ed.), Nueva historia de Colombia (Vol. II) (pp. 295-340). Bogotá: Planeta.

Villegas, J. y Yunis, J. (1978). La Guerra de los Mil Días. Bogotá: Carlos Valencia Editores.

Wilde, A. (1982). Conversaciones de caballeros: la quiebra de la democracia en Colombia. Bogotá: Ediciones Tercer Mundo.

Zambrano, F. (1998). El golpe de Melo de 1854. En F. Zuluaga Ramírez et al., Las guerras civiles desde 1830 y su proyección en el siglo XX: Memorias (pp. 60-72). Bogotá: Museo Nacional de Colombia.

Zuluaga, F. (1998). La Guerra de los Supremos en el suroccidente de la Nueva Granada. En F. Zuluaga Ramírez et al., Las guerras civiles desde 1830 y su proyección en el siglo XX: Memorias (pp. 17-36). Bogotá: Museo Nacional de Colombia. 